\title{
Microfluidic Techniques for Circulating Tumour Cells Separation
}

\author{
Jessica N Sierra-Agudelo ${ }^{1 *}$, Lluís Figueras ${ }^{1}$, Miguel Mir ${ }^{1}$, Roberto Paoli ${ }^{1}$, Romen Rodríguez-Trujillo ${ }^{1,2}$, \\ Josep Samitier ${ }^{1,2}$ \\ ${ }^{1}$ Nanobioengineering Group, Institute for bioengineering of Catalonia (IBEC) \\ c/Baldiri Reixac 10-12, 08028 Barcelona, Spain \\ jsierra@ibecbarcelona.eu \\ ${ }^{2}$ Department of Electronics and Biomedical Engineering, University of Barcelona (UB) \\ C/ Martí I Franquès 1, 08028 Barcelona, Spain
}

\section{Extended Abstract}

Liquid biopsy has become a promising technique for early cancer detection, molecular stratification, detecting treatment relapse, monitoring treatment response and tumor evolution, as well as establishing a personalized treatment program. Currently, this technique is based on the analysis of circulating tumor cells (CTCs), circulating tumor DNA (ctDNA), or tumor-derived extracellular vesicles present in blood [1].

The possibility to process small blood samples represent an excellent approach to monitor the disease course without obtaining tissue directly from the tumor. The alternative results in lower costs with a non-invasive process and the possibility to detect the condition in earlier stages, which would have a great incidence in morbidity rates. Nevertheless, one of the most relevant challenge in this field involves the processing and analyzing of CTCs, due to their low amount in peripheral blood (1 to 100 CTCs per $10^{9}$ blood cells) [2]. Thus, a highly specialized enrichment method is necessary to harvest high-purity and viable CTCs suitable for subsequent molecular analysis.

Nowadays, the approaches for isolating CTCs from blood samples are limited due to high cell contamination rates or substantial loss of cancer cells, and high cost methods. In order to overcome these limitations, microfluidic devices have been designed for isolating CTCs based on their intrinsic properties like density, size, deformability and difference in membrane protein expression [3].

Based on the properties mentioned above, we developed lab-on-a-chip (LOC) platforms using different fabrication techniques such as soft lithography and 3D-printing. The devices combine hydrodynamic sorting, inertial forces and/or cell deformability based on differences in young modulus values between normal blood cells and CTCs. In our method, the use of hydrodynamic sorting can efficiently divide target cells and other cells into different groups by size and guide their movement in their respective trajectories [4]. For developing our CTCs isolation system, we first manufactured a simple microfluidic device composed by two different inlets, one of them use for the blood sample and another one for the focusing liquid (phosphate buffered saline). Our preliminary results revealed that the device can efficiently focus CTCs and separate them from most of the blood cells. Indeed, experiments performed with whole blood samples from healthy donors and polystyrene particles of $30 \mu \mathrm{m}$ as a CTCs model showed that the particles were correctly recovered (100\%), with a very high red blood cell depletion (99.3\%). Depletion of white blood cells, however, was not as high (87\%) due to the inherent overlap in size with CTCs.

In order to overcome the limitations of the previous device, we manufactured a second system designed to capture the remaining leukocytes using an affinity-binding principle. The device includes a herringbone structure with microfabricated ridges placed on the roof of the channel [5]. These structures produce a transverse component in the flow, subsequently helical streamlines are generated, and an increase in the surface interaction with cells takes place. One of the most relevant features of this device is the surface modification with a Self-assembled monolayer (Biotin-PEG-thiol) and a Biotin-PEG$\mathrm{OH}$ as an additional blocking agent in the chip surface. Thus, CD45-antibody is immobilized in the inner channel surface to capture Leucocytes and obtaining a high purity CTC sample. This kind of surface modification has several advantages, such as, a better antibody orientation, homogeneous antibody distribution in the surface and long-term stability [6].

In conclusion, we have developed a set of microfluidic devices that, based on hydrodynamic inertial effects are capable of isolating circulant tumor cells from high concentrated blood samples. The devices are fabricated from polymeric 
biocompatible materials and using low-cost techniques. The proposed devices will pave the way to the development of lowcost compact diagnostic systems for early cancer detection.

\section{References}

[1] H. W. Hou, M. E Warkiani, B. L. Khoo, Z. R. Li, R. A. Soo, D. S. Tan, W. Lim, J. Han, A. A. S. Bhagat and C. T. Lim, "Isolation and retrieval of circulating tumor cells using centrifugal forces," Scientific Reports, vol. 3, no. 1, 2013.

[2] Y. Deng, Y. Zhang, S.Sun, Z. Wang, M. Wang, B. YU, D. M. Czajkowsky, B. Liu, Y. LI, W. Y Wei and Q. Shi, "An Integrated Microfluidic Chip System for Single-Cell Secretion Profiling of Rare Circulating Tumor Cells," Scientific Reports, vol. 4, no. 1, 2014.

[3] Y. Chen, P. Li, P. Huang, Y. Xie , J. D. Mai, L. Wang, N. Nguyen and Y. Huang, "Rare cell isolation and analysis in microfluidics," Lab on a Chip, vol. 14, no. 4, p. 626, 2014

[4] M. E. Warkiani, B. L. Khoo, L. Wu, A. K. P. Tay, A. A. S. Bhagat, J. Y Han, C. T. Lim, "Ultra-fast, label-free isolation of circulating tumor cells from blood using spiral microfluidics," Nature Protocols, vol. 11, no. 1, pp. 134$148,2015$.

[5] S. L. Stott, C. Hsu, D. I. Tsukrov, M. Yu, D. T. Miyamoto, B. A. Waltman, S. M. Rothenberg, A. M.Shah, M. E. Smas, G. K. Korir, F. P. Floyd, A. J. Gilman, J. B. Lord, D. Winokur, S. Springer, D. Irimia, S. Nagrath, L. V. Sequist, R. J. Lee, K. J. Isselbacher, S. Maheswaran, D. A. Haber and M.Toner, "Isolation of circulating tumor cells using a microvortex-generating herringbone-chip," Proceedings of the National Academy of Sciences, vol. 107, no. 43, pp. 18392-18397, 2010. 